\title{
Spray Dried Micronized Particles and Electrostatic Separation
}

\author{
A. Justen*, G. Schaldach, M. Thommes \\ Laboratory of Solids Process Engineering, TU Dortmund University, Dortmund, Germany \\ ${ }^{*}$ Correspondence: anna.justen@tu-dortmund.de
}

\begin{abstract}
Low aqueous solubility of drug substances is still one major challenge in pharmaceutical development. A general approach is the reduction of drug particle size to increase dissolution rate as well as solubility. Spray drying has been proposed as appropriate continuous manufacturing technique for micronized particles, which means a median diameter of less than $10 \mu \mathrm{m}$.

For the production of sub-micron particles by spray drying small droplets are necessary and therefore an ultrasonic nebulizer operating at a frequency of $3 \mathrm{MHz}$ and suitable for acetone is used.

In this study a new apparatus was designed and the process parameters were determined. The aerosol consists of fine droplets of dissolved drug in acetone and is transported in carbon dioxide saturated with acetone. After evaporation the acetone vapour is removed by condensation. The resulting dry drug particles are separated from the carbon dioxide gas stream by electrostatic precipitation. The product has been analysed with laser diffraction method. Micronized drug particles were obtained at various process conditions.

The presented technique was found to be appropriate for continuous manufacturing of micronized particles. The particles have been investigated regarding the particle size and shape.
\end{abstract}

\section{Keywords}

spray drying, micronized particles, organic solvent, ultrasonic atomization, laser diffraction

\section{Introduction}

The low aqueous solubility of novel drug molecules is one of the main challenges in drug formulation these days [1]. One approach to overcome this is the reduction of particle size as it leads according to Nernst and Brunner [2] to an enhanced dissolution rate. It is also known that a reduced particle size increases the solubility according to Ostwald and Freundlich [3]. Recently, spray drying has been suggested as manufacturing technique for micronized particles [4-6].

Several techniques have been used for the generation of micronized particles such as pneumatic two- fluid nozzles [4, 7], electro spraying [8] or ultrasonic atomization [9] with vibrating mesh technique $[10,11]$.

Advantages of droplet formation with ultrasonic atomizers are the uniform size distribution, a high sphericity of the emerging droplets, as well as a low liquid pressure [12].

In order to obtain small particles the generation of small droplets is required. Compared to conventional spray dryers like the Nano Spray Dryer B-90 by Buechi (Buechi Labortechnik, Flawil, Switzerland) or the ProCepT (Zelzate, Belgium), where frequencies of up to $140 \mathrm{kHz}$ are applied $[13,14]$, the ultrasonic atomizer in this study operates at a particularly high frequency of $3 \mathrm{MHz}$.

In this study a custom-made spray drying apparatus operating with carbon dioxide, including the removal of the organic solvent for continuous particle generation, was designed. 
Appropriate process parameters for the production of micronized particles were determined and the product was deposited in an electrostatic precipitator. The aim of this study was the development and characterization of a spray drying process with the new designed aerosol generator.

\section{Material and Methods}

A solution of acetone and an active pharmaceutical ingredient (API) was dispersed with an ultrasonic atomizer (WHQ 3005/1530-12N, Siansonic Technology, Beijing, China) which is operating at a particularly high frequency of $3 \mathrm{MHz}$.

Phenytoin was used as model substance. Due to the high solubility of phenytoin in acetone (33 g/L) [15] a high throughput was expected.

The fine droplets were transported into the evaporation and the condensation stage with carbon dioxide as transporting gas. Finally, the dry particles were collected in an electrostatic precipitator (ESP), which has been described in a previous study [16]. For the operation of an ESP in combination with organic solvents the application of carbon dioxide becomes indispensable.

Particle and droplet size distribution was analysed with laser diffraction (Spraytec, Malvern Panalytical, Malvern, UK). The droplet size was measured at the outlet of the aerosol chamber, the particle size was measured right after the condensation stage. Mie theory (light scattering) was used for evaluation, while a $300 \mathrm{~mm}$ lens was utilized. The repeated measurements of $10 \mathrm{~s}$ were conducted and the average particle and droplet size was calculated.

The 12 inner detectors were disabled due to an interference with the carrier gas. This was possible since there were no large particles in the aerosol.

The particle morphology of the spray dried particles was assessed with scanning electron microscopy (SEM). The gas-borne particles were collected on a glass surface after drying before entering the MESP. The SEM images (H-S4500 FEG, Hitachi, Krefeld, Germany) were taken at $1 \mathrm{kV}$ without any additional sample preparation.

\section{Results and Discussion}

In this study, an aerosol generator for the production of micronized drug particles was designed and suitable process parameters were determined.

The spray drying apparatus can be divided into different unit operations (see Figure 1).

For material supply, an organic phenytoin solution is fed into an aerosol chamber, where it is nebulized. The aerosol is transported with a carbon dioxide gas stream into a drying stage. The drying stage consists of an evaporation and a condensation section. Therefore fine particles leave the aerosol generator whereby the evaporated organic solvent is removed from the gas stream. The particles are collected for further processing or investigation in the separation stage, which is designed as electrostatic precipitator. 


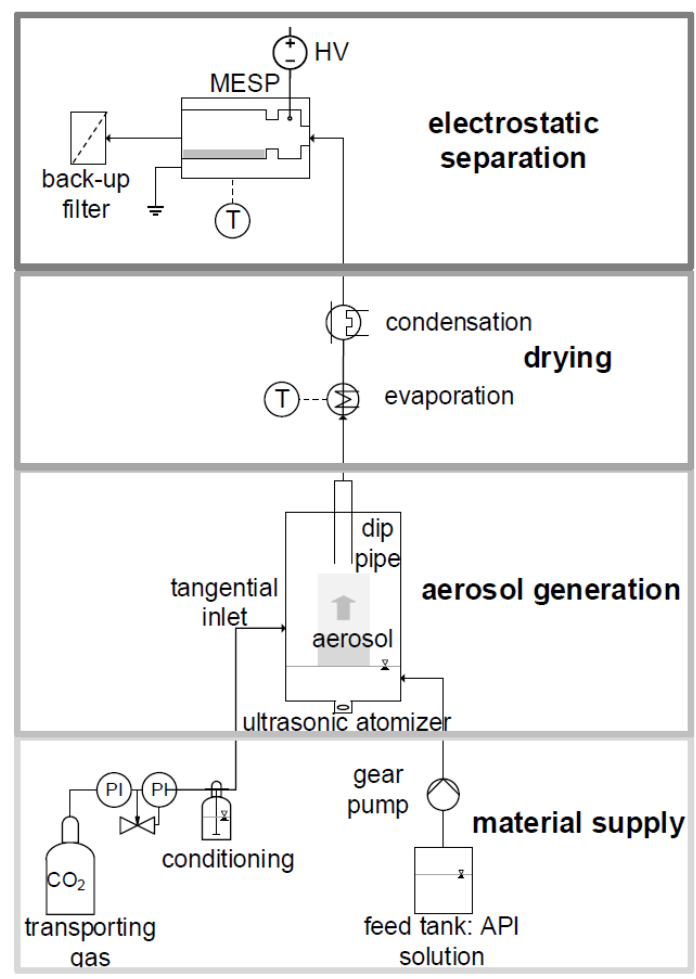

Figure 1: schematic of the experimental set-up

One main part of the investigated apparatus is the aerosol generation in the aerosol chamber (see Figure 2). It has a volume of about $220 \mathrm{~mL}$, with an inlet for the liquid and gas supply.

The API solution is pumped into the aerosol chamber, where it is nebulized by ultrasonic atomization. The ultrasonic atomizer is placed at the bottom of the aerosol chamber (see Figure 2) where it is overflown with the API solution. It was chosen based on its high excitation frequency of $3 \mathrm{MHz}$. The droplet size mainly depends on the physico-chemical properties of the atomized liquid as well as the excitation frequency as below [17]:

$d_{p}=0.34\left(\frac{8 \pi \sigma}{\rho f^{2}}\right)^{\frac{1}{3}}$

According to the material properties of acetone [18] a droplet size $1.4 \mu \mathrm{m}$ can be estimated. This equation is one approximation to predict the droplet size, but properties like viscosity of the fluid and liquid flow rate are neglected here. Many effort has been made to predict the droplet size in ultrasonic atomizers lately $[12,19]$. As a rough estimation Lang's equation should be regarded as a sufficient prediction here. 




Figure 2: aerosol chamber with ultrasonic atomizer

The API solution is pumped into the aerosol chamber continuously during the nebulizing process resulting in a constant film thickness above the piezo ceramic. The pump rate corresponds to the atomizer performance of $5 \mathrm{~mL} / \mathrm{min}$. The film thickness is thereby kept constant at $25 \mathrm{~mm}$, which was found to be the operating optimum.

Based on the concentration of the API solution and the atomizer performance a particle mass flow of $7.5 \mathrm{~g} \mathrm{~h}^{-1}$ can be expected.

Carbon dioxide is supplied to the process via a tangential inlet of the aerosol chamber. It serves as both transporting and drying gas. Thereby the gas volume flow can be adjusted to the requirements of the process and the droplet generation is independent of the gas feed, compared to pneumatic or pressure nozzles.

The atomized droplets are transported upwards into the drying unit. Because of the tangential inlet, a vortex emerges so that larger droplets are directed against the wall. These droplets are led to the ground of the aerosol chamber where they merge with the film above the piezo ceramic and can once again be atomized.

The gas borne particles are transported upwards through the dip pipe. The slowest gas volume flow, which results in a vortex, was determined to be $4.9 \mathrm{~L} \mathrm{~min}{ }^{-1}$. It was set as gas volume flow throughout the study in order to obtain possibly small particles. Additionally a low volume flow rate leads to a high residence time of the particles in the separation stage, which increases the separation efficiency in the electrostatic precipitator.

The evaporation stage consists of a glass pipe of $400 \mathrm{~mm}$ length, which is wrapped with a heating band. The temperature of the heating band is controlled by the gas temperature at the end of the evaporation stage and is adjusted throughout the process to $80^{\circ} \mathrm{C}$.

The condensation stage consists of an intensive cooling pipe, in order to remove acetone from the process. Thereby an acetone free gas stream accesses the separation stage.

Droplet and particle size distribution was investigated using laser diffraction analysis. Droplet sizes were measured at the top of the aerosol chamber, right before the evaporation stage, while particles were detected after evaporation and condensation of the solvent.

Bimodal droplet and particle size distributions were found (see Figure 3). 


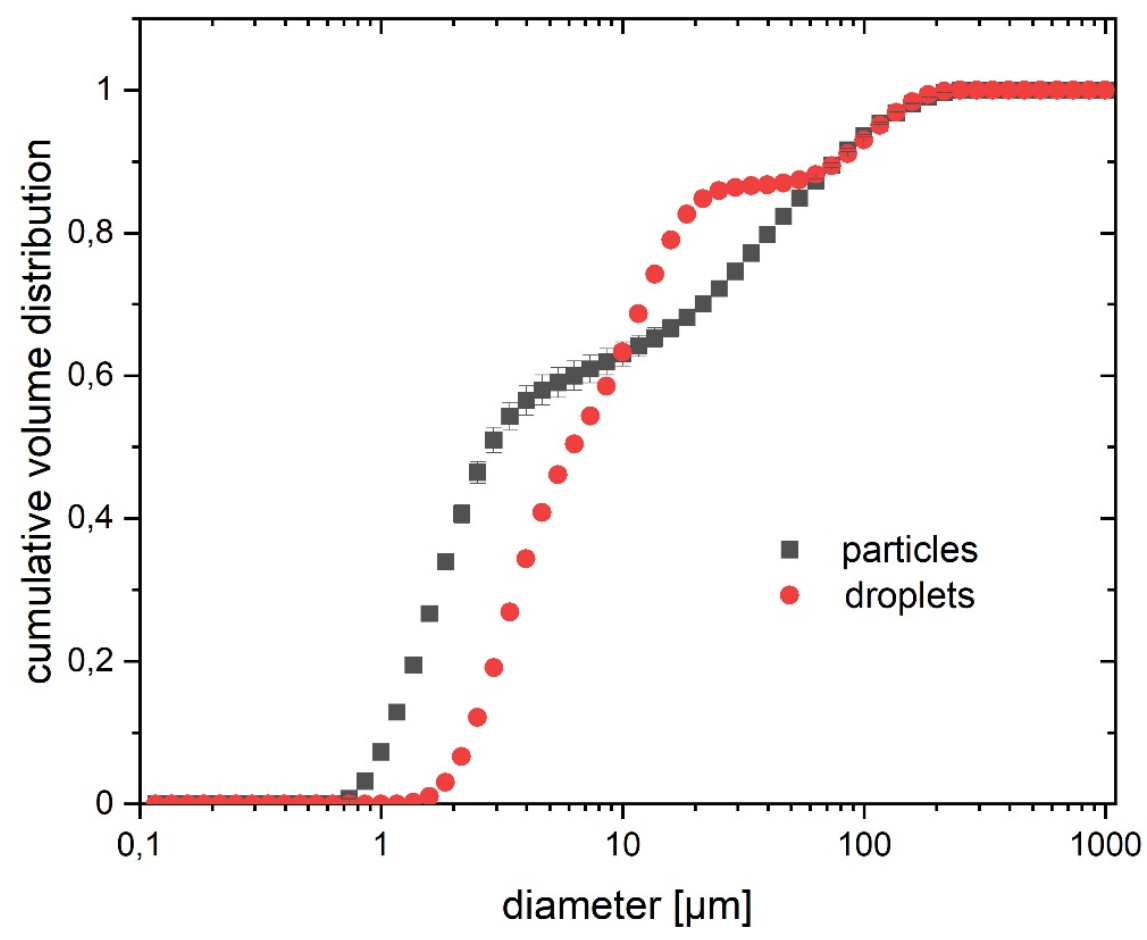

Figure 3: volume distribution of atomized droplets (red circle) of spray dried particles (black square); av $\pm s, n=3$

The droplets as well as the dry particles show bimodal size distributions. Besides the small droplets of less than $10 \mu \mathrm{m}$ larger droplets of more than $50 \mu \mathrm{m}$ are seen as well. However the volume fraction of the desired particles is rather high $(80 \%)$, which confirms the concept of ultrasonic atomization to be appropriate. The droplet mass flow is about $211.7 \mathrm{~g} / \mathrm{h}$, which is rather high with respect to alternative dispersing techniques.

The bimodal size distribution of the droplets can be seen for the particles as well. This is expected. However, there is a change in size distribution that might be related to different drying kinetics of small and large droplets. This would change the volume ratio if large particles are more porous or hollow. A volume fraction of particles of about $60 \%$ is close to the desired micronized range.

First measurements dealt with evaluation of particle size and shape immediately after aerosol generation and drying. Scanning Electron Microscope images were taken and a representative one is given in Figure 4. The rectangular and rhombic particle shape indicates a crystalline state as described in the literature [20]. The particle size is in the desired micronized range (see Figure 4) and consistent with the particle size distribution (Figure 3) considering the difference of number and volume distribution. 


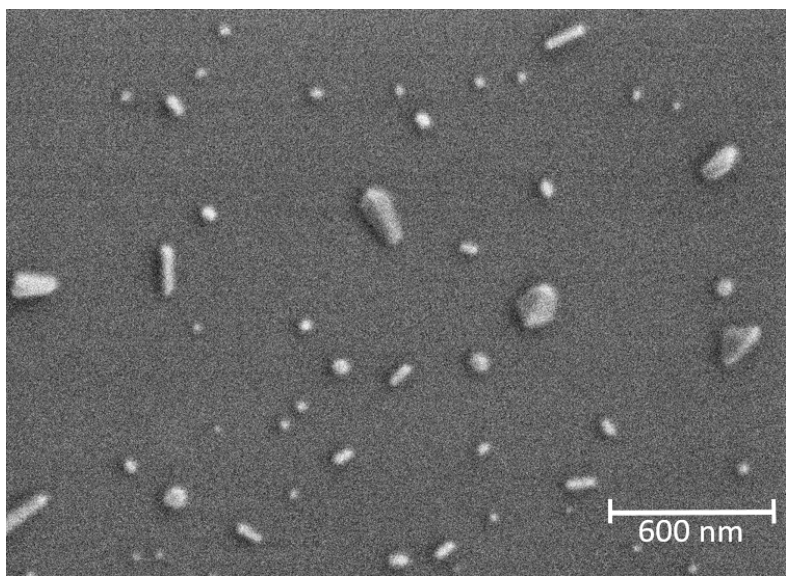

Figure 4: SEM Image of spray dried phenytoin particles

\section{Conclusions}

In this study, an experimental setup for spray drying of micronized particles was designed, built and tested. Thereby a new aerosol generator was implemented using a piezo electric ceramic optimized for a particularly high resonance frequency. It was possible to produce micronized drug particles which will be investigated in further studies. Process parameters were determined and a first characterization of the product was conducted. An effect of carbon dioxide on the detection quality of laser diffraction was known before. Interferences caused by local differences in the refraction index or temperature are known disturbances, but can normally be overcome by disabling the inner detectors of the laser diffraction system.

The expected droplet and particles sizes have been estimated according to the maximum gas bound diameter.

\section{Acknowledgments}

This project was made possible by the funding of European Regional Development Fund (EFRE).

\section{Nomenclature}

$d_{p} \quad$ droplet diameter [m]

$\sigma \quad$ surface tension $\left[\mathrm{N} \mathrm{m}^{-1}\right]$

$\rho \quad$ density of the liquid $\left[\mathrm{kg} \mathrm{m}^{-3}\right]$

$f \quad$ excitation frequency $[\mathrm{Hz}]$

\section{References}

[1] Lipinski, C. A., 2000, "Changes in the profiles of drug properties: An experimental, computational, and informatics perspective.," Abstr Pap Am Chem S, 219, pp. U459-U459.

[2] Nernst, W., and Brunner, E., 1904, "Velocity of Reaction in Non-Homogeneous System," Physical Chemistry, 47, pp. 56-102.

[3] Junghanns, J. U., and Muller, R. H., 2008, "Nanocrystal technology, drug delivery and clinical applications," Int J Nanomedicine, 3(3), pp. 295-309.

[4] Strob, R., Dobrowolski, A., Schaldach, G., Walzel, P., and Thommes, M., 2018, "Preparation of spray dried micronized particles: Part A - Particle generation by aerosol conditioning," Int J Pharm, 548(1), pp. 423-430.

[5] Li, X., Anton, N., Arpagaus, C., Belleteix, F., and Vandamme, T. F., 2010, "Nanoparticles by spray drying using innovative new technology: the Buchi nano spray dryer B-90," J Control Release, 147(2), pp. 304-310.

[6] Dobrowolski, A., Strob, R., Drager-Gillessen, J. F., Pieloth, D., Schaldach, G., Wiggers, H., and Thommes, M., 2019, "Preparation of micronized drug particles via spray drying from organic solvents," Int J Pharm, 567, p. 118501.

[7] Dobrowolski, A., Strob, R., Nietfeld, J., Pieloth, D., Wiggers, H., and Thommes, M., 2018, "Preparation of spray dried micronized particles: Part B - Particle recovery by electrostatic precipitation," Int J Pharm, 548(1), pp. 237243. 
[8] Smeets, A., Clasen, C., and Van den Mooter, G., 2017, "Electrospraying of polymer solutions: Study of formulation and process parameters," Eur J Pharm Biopharm, 119, pp. 114-124.

[9] Bittner, B., and Kissel, T., 1999, "Ultrasonic atomization for spray drying: a versatile technique for the preparation of protein loaded biodegradable microspheres," J Microencapsul, 16(3), pp. 325-341.

[10] Arpagaus, C., John, P., Collenberg, A., and Rutti, D., 2017, "Nanocapsules formation by nano spray drying," Nanoencapsulation Technologies for the Food and Nutraceutical Industries, pp. 346-401.

[11] Durli, T. L., Dimer, F. A., Fontana, M. C., Pohlmann, A. R., Beck, R. C., and Guterres, S. S., 2014, "Innovative approach to produce micronized drug particles by vibrational atomization spray drying: influence of the type of solvent and surfactant," Drug Dev Ind Pharm, 40(8), pp. 1011-1020.

[12] Rajan, R., and Pandit, A. B., 2001, "Correlations to predict droplet size in ultrasonic atomisation," Ultrasonics, 39(4), pp. 235-255.

[13] Schmid, K., Arpagaus, C., and Friess, W., 2011, "Evaluation of the Nano Spray Dryer B-90 for pharmaceutical applications," Pharm Dev Technol, 16(4), pp. 287-294.

[14] Ormes, J. D., Zhang, D., Chen, A. M., Hou, S., Krueger, D., Nelson, T., and Templeton, A., 2013, "Design of experiments utilization to map the processing capabilities of a micro-spray dryer: particle design and throughput optimization in support of drug discovery," Pharm Dev Technol, 18(1), pp. 121-129.

[15] NCBI, Accessed on 25 Februrary 2021, "Phenytoin," Available online: https://pubchem.ncbi.nlm.nih.gov/compound/Phenytoin\#section=Solubility.

[16] Dobrowolski, A., Pieloth, D., Wiggers, H., and Thommes, M., 2019, "Electrostatic Precipitation of Micronized Particles in a Molten Carrier," Pharmaceutics, 11(6).

[17] Lang, R. J., 1962, "Ultrasonic atomization of liquids," The journal of the acoustical society of America, 34(1), pp. 6-8.

[18] NCBI, Accessed on 25 February 2021, "Acetone," Available online: https://pubchem.ncbi.nlm.nih.gov/compound/Acetone\#section=Density.

[19] Walzel, P., 1993, "Liquid atomization," International Chemical Engineering (A Quarterly Journal of Translations from Russia, Eastern Europe and Asia);(United States), 33(1).

[20] Nokhodchi, A., Bolourtchian, N., and Dinarvand, R., 2003, "Crystal modification of phenytoin using different solvents and crystallization conditions," Int J Pharm, 250(1), pp. 85-97. 